\title{
Field Evaluation of Yield and Yield Component Traits of Breeding Lines of Maize over Two Seasons in Derived Savannah Agro-Ecology
}

\author{
Charles U. UBA ${ }^{1 *}$, Christian U. AGBO ${ }^{1}$, Uchechukwu P. CHUKWUDI ${ }^{1}$, \\ Andrew A. EFUSIE ${ }^{2}$, Stella O. MUOJIAMA ${ }^{1}$ \\ ${ }^{1}$ University of Nigeria, Department of Crop Sciencem Nsukka, Enugu State, Nigeria; charlesuba192@gmail.com ("corresponding authors); \\ christian.agbo@unn.edu.ng;uchechukwu.chukwudi@unn.edu.ng; stellypotential@yahoo.com \\ ${ }^{2}$ University of Port Harcourt, Department of Crop \& Soil Science, P.M.B. 5323 Choba, Port Harcourt, Rivers State, \\ Nigeria; andyefisue@yahoo.com
}

\begin{abstract}
The understanding of yield and the interaction with its components is very important for selection in early generations of crop breeding. Twelve maize genotypes were collected from International Institute for Tropical Agriculture (IITA) along with seven landraces in order to identify the contribution of different traits to yield improvement. The experiments were carried out in two different seasons (March/April-early and July/August- late) in a randomized complete block design with three replications. Early season planting had a higher grain yield than late season planting. The difference in grain yield between early and late season was 3.92 tons/ha. This represents a $27.8 \%$ increase in grain yield during the early season over the late season planting. Number of ears per plant and shelling percentage were not influenced by seasonal effects. Ear weight and days to tasselling showed the highest direct positive effects of 0.972 and 0.665 , respectively on grain yield, during early season. Furthermore, ear weight, followed by shelling percentage, exerted the highest direct positive effect on grain yield in late season. Higher indirect positive effects were obtained for ear diameter, ear length, ear height and plant height via ear weight in both seasons. Ear weight, days to tasselling and ear length were identified as the major traits affecting yield of maize in both seasons in the derived Savannah agro-ecology.
\end{abstract}

Keywords: contrasting season; correlation coefficient; path coefficient analysis; yield; Zea mays

\section{Introduction}

Maize (Zea mays L) belongs to the family of grass Poaceae. It is an important staple food crop produced in many countries in sub-Sahara Africa. Maize is among the leading cereal crops, after wheat and rice, with regards to its cultivation areas, production and consumption (Olakojo and Akinlosotu, 2004; Olawuyi et al., 2010). It is also a versatile crop grown across a wide range of ecological zones (Akinbode, 2010; Bello et al., 2012). Maize serves as feed for livestock, raw materials for pharmaceutical and many agroallied industries in the world (Obi, 2006).

In spite of the importance of maize to Nigerians and their economy, its production has not been able to meet the demand of the teeming population because it is affected by environmental condition caused by climate change (Ibrahim et al., 2014). Climate variability conditioned by global warming has affected temperature, rainfall distribution and other climatic factors. The relationship between climate and growing season parameters (onset and cessation of rains), has made the growing season variability an important indicator of climate change (Linderholm et al., 2008). Understanding the various management practices, as well as the environmental conditions that affect crop performance, is required for successful maize production (Eckert, 1995). Analyzing genotype performance under field condition is a necessity in understanding environmental effects on crop growth at various planting seasons and the ultimate performance of a cultivar. Selection of appropriate cultivars, planting dates and plant densities are cultural practices that have shown to affect maize yield potential and stability (Norwood, 2001). Path-coefficient analysis is a useful tool for identifying indirect selection criteria for complex quantitative traits in crop program.

Grain yield is a quantitative trait controlled by many genes of minor effects and highly influenced by environmental fluctuations. Understanding the relationship existing between grain yield and other metric traits is important for maize improvement. Rani et al. (2017) identified that the number of grains per row, as well as 1,000 grain weight, cob diameter and plant height are useful characters in a maize breeding program. Tyagi and Khan 
568

(2010) stressed location specific trial in maize improvement due to environmental effect on maize yield.

Therefore, it is important to evaluate maize genotypes in various agro-ecological zones for yield potential, adaptation, pest and disease reaction, other stress factors, to identify genotypes that can replace the existing cultivars, as part of requirement for releasing suitable varieties to farmers for cultivation (Olakojo and Iken, 2001). Furthermore, it is a necessity that every genotype before it is introduced to a new agro-ecology should be assessed for yield potential and other environmental stress factors, before it is recommended for cultivation, because every agro-ecology has its unique growing conditions. Hence, the objectives of the present study were to evaluate the yield performance of new and existing maize genotypes in a derived Savannah agro-ecology, determine their best planting season and identify traits that imparted most for selection.

\section{Materials and Methods}

The field experiments were carried out in the Research Farm of the Department of Crop Science, Faculty of Agriculture, University of Nigeria, Nsukka. Nsukka is located at latitude $06^{\circ} 51^{\prime} \mathrm{N}$, longitude $07^{\circ} 29^{\prime} \mathrm{E}$ and altitude $400 \mathrm{~m}$ above sea level. It is characterized by low land humid condition with bimodal annual rainfall distribution that ranges from $1,155 \mathrm{~mm}$ to $1,955 \mathrm{~mm}$, a mean annual temperature of $29{ }^{\circ} \mathrm{C}$ to $31{ }^{\circ} \mathrm{C}$ and relative humidity that ranges from $69 \%$ to $79 \%$ (Uguru et al., 2011). The experiment was carried out in the year 2014 in early (March/April) and late (July/August) planting season which represent the common planting period for early and late maize in this agro-ecology, respectively.

Nineteen open pollinated maize genotypes (Table 1) were used in the study. Twelve of the genotypes were new in this derived Savannah agro-ecology and were collected from International Institute for Tropical Agriculture, Ibadan, Nigeria, while the remaining seven are landraces evaluated for the two seasons.

The meteorological data for the duration of the experiment were collected from the Department of Crop Science meteorological station (Table 2). The plots measured $2 \times 5 \mathrm{~m}$ with plant spacing of $75 \mathrm{~cm}$ inter row and $25 \mathrm{~cm}$ intra row. A distance of $1 \mathrm{~m}$ between plots and blocks was maintained. This was to ensure that there was no shading effect of tall genotypes over short genotypes. Two seeds per hill were planted, which were later thinned to one plant per hill, at 4-5 leaf stage. The plant density was 53,333 plants/ha. Two independent experiments per season were laid out in randomized complete block design with three replications. First application of fertilizer was at two weeks after planting (NPK 20:10:10) at a rate of $100 \mathrm{~kg} / \mathrm{ha}$, while the second treatment was with $75 \mathrm{~kg} / \mathrm{ha}$ urea at tasselling stage. Weeding was done 3 times manually. Data were collected from the middle rows of each plot, except stem borer incidence, number of ears per plant, days to $50 \%$ tasselling and silking.

The parameters measured were days to $50 \%$ tasselling, days to $50 \%$ silking, plant height at maturity, ear height, number of ears per plant, ear length, ear diameter, shelling percentage, hundred grain weight, stem borer incidence, number of exit hole, tunnel damage length and grain yield. Number of ears per plant was estimated by counting the total number of ear harvested in the plot divided by the total number of plants in the plot. Days to $50 \%$ tasselling and silking were estimated as number of days from planting to the date that tasselling and silking started in $50 \%$ of the plants in a plot. Percentage incidence of stem borers on each plot was determined by counting the number of plants with stem borer infestation divided by the total number of plants, multiplied by one hundred, according to Obi (1981). At early planting season, number of exit hole and tunnel damage length were absent on the maize stems, but present at latter season plants.

Mathematically, the following formula was used:

Stemborer incidence $=$ (number of plants with stem borer infestation per plant/ Total number of plants per plot) $\times 100$

Grain yield (kg/plot): harvested ears were threshed and the grains weighed per plot. The grain weight was later converted to tonnes per hectare ( $t / h a)$ after adjusting for seed moisture content of $15 \%$ at harvest.

Yield difference was calculated mathematically as SHY SLY:

Percentage yield $=($ SHY-SLY $/$ SBS $) \times 100$

Where SHY = Season with higher yield, SLY = Season with lower yield, $\mathrm{SBS}=$ Sum of both season

Statistical analysis

Data obtained in each season were subjected to analysis of variance (ANOVA) separately, before combined ANOVA for the early and late season was done, using GenStat software to compute mean squares for each trait. Differences in traits means were compared using Least Significant Difference (LSD). Pearson correlations analysis was performed using Statistical Package for Social Science (SPSS 16) to examine inter-relationship among the traits in each season. The sets of correlation coefficients were subjected to path coefficient analysis and the direct and indirect effects of each season using the method proposed by Wright (1934) and the techniques of Dewey and Lu (1959).

In the path diagram (Fig. 1), single-arrowhead lines represent the direct effects of a predictor variable on its response variable, while double-arrowhead lines represent the mutual association between variables as measured by correlations. The variable residual $(\mathrm{X})$ is the underdetermined portion (i.e. sampling error and those variables not included in the model).

\section{Results}

The meteorological results at the experimental site revealed that the rainfall distribution followed a bimodal pattern of rainfall (Table 2). The first and second peak of rainfall was in June and September, respectively. September had the highest peak of rainfall $(401.99 \mathrm{~mm})$ and a drop in August (August break). Maximum temperature was recorded in April $\left(31.30^{\circ} \mathrm{C}\right)$, while August and September recorded the highest relative humidity of $73 \%$. 


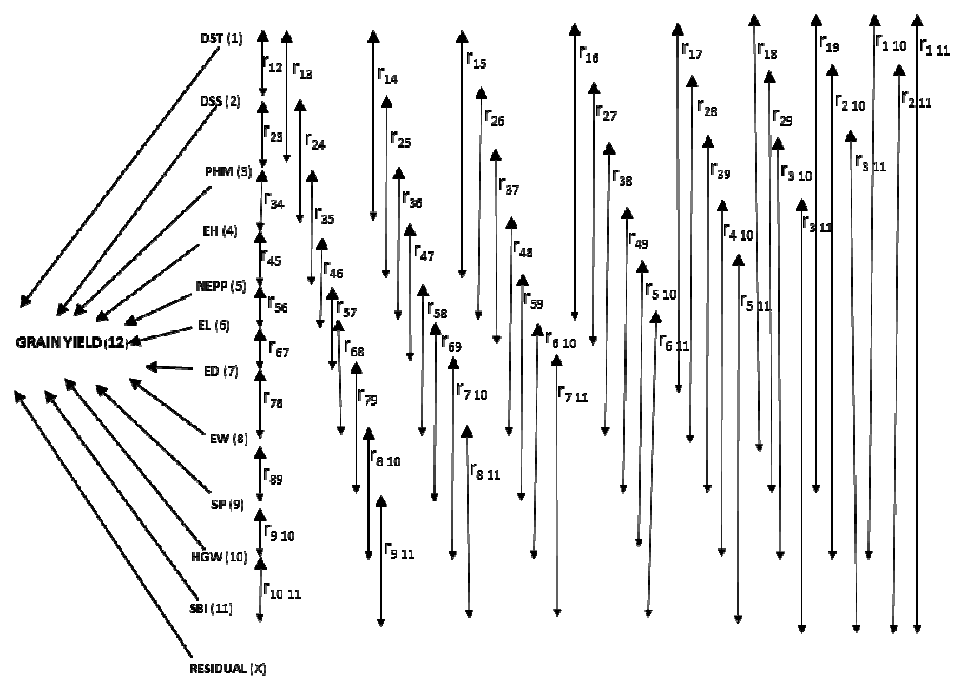

Fig. 1. Path diagram showing causal relationship between the eleven predictor variables: Days to tasselling (DST), Days to silking (DSS), Ear height (EH), Plant height at maturity (PHM), Ear Diameter (ED), Ear length (EL), Number of ears per plant (NEPP), Ear weight (EW), Shelling percentage (SP), Hundred grain weight (HGW), Stem borer incidence (SBI) and the response variable (i.e. grain yield) for both seasons. The variable residual $(\mathrm{X})$ is the underdetermined portion

Table 1. Maize genotypes studied in the experiment

\begin{tabular}{cccc}
\hline S/N & Genotypes & Source of seed collection & Grain colour \\
\hline 1 & '2009 TZE OR1 DT STR QPM' & IITA & Yellow \\
\hline 2 & '2009 TZE OR2 DT STR QPM' & IITA & Yellow \\
3 & 'AMA TZBR - Y - F2' & IITA & Yellow \\
4 & 'TZBR COMP - YC1F2' & IITA & Yellow \\
5 & 'TZBR COMP - - YC1F2' & IITA & Yellow \\
6 & 'DMR - LSR - Y' & IITA & Yellow \\
7 & 'BR 9928 DMRSR' & IITA & White \\
8 & 'BR 9943 DMRSR' & IITA & Yellow \\
9 & 'TZE - Y - POP DT STR QPM' & Yellow \\
10 & '99 TZE Y - STR QPM' & IITA & Yellow \\
11 & 'TZEE - Y -PORSTR QPM CO' & Yellow \\
12 & 'EV DT - Y - 2000 STR QPM' & IITA & Yellow \\
13 & 'UGWUACHARA' (landrace) & Yellow \\
14 & 'UMUKASI' (landrace) & Nsukka L.G.A & Yellow \\
15 & 'ORBAl' (landrace) & Nsukka L.G.A & Yellow \\
16 & 'ORBA2' (landrace) & Udenu L.G.A & Yellow \\
17 & 'OBUKPA' (landrace) & Udenu L.G.A & Yellow \\
\hline 18 & 'EDEM ANI' (landrace) & Nsukka L.G.A & Yellow \\
\hline
\end{tabular}

IITA = International Institute of Tropical Agriculture, L.G.A = Local Government Area

Table 2. Metrological data for the study period

\begin{tabular}{cccccc}
\hline \multirow{2}{*}{ Month } & \multicolumn{2}{c}{ Temperature $\left({ }^{\circ} \mathrm{C}\right)$} & \multicolumn{2}{c}{ Relative humidity $(\%)$} \\
\cline { 2 - 6 } & Rainfall $(\mathrm{mm})$ & Minimum & Maximum & 10.00 am & $4.00 \mathrm{pm}$ \\
\hline March & 14.23 & 22.52 & 31.71 & 62.77 & 65.55 \\
April & 105.16 & 22.30 & 31.30 & 69.93 & 70.53 \\
May & 241.14 & 21.06 & 28.29 & 72.26 & 72.26 \\
June & 271.79 & 20.87 & 29.13 & 72.00 & 72.00 \\
July & 195.81 & 20.90 & 27.74 & 72.19 & 72.19 \\
August & 92.36 & 20.71 & 27.29 & 73.00 & 73.00 \\
September & 401.99 & 20.33 & 27.90 & 73.00 & 73.00 \\
October & 211.08 & 20.84 & 28.90 & 73.00 & 72.77 \\
November & 77.22 & 21.00 & 30.07 & 73.80 & 71.97 \\
\hline Mean & 179.00 & 21.17 & 29.08 & 72.44 & 1.98 \\
\hline FLSD(0.05) & 25.19 & NS & 0.96 & NS & 1.98 \\
\hline
\end{tabular}


570

Genotypic performance of agronomic and yield component traits

The agronomic traits of the maize genotypes differed significantly $(\mathrm{p}<0.05)$ during the two seasons (Table 3$)$. Among the genotypes 'TZE - Y -POR STR QPM CO' had significantly shorter days to tasselling and silking than the other genotypes, except for 'TZE - Y - POP DT STR QPM'. Maize genotypes at early season planting attained flowering earlier, with taller ear height and plant height when compared to late season planting genotypes. The genotype 'Orba2' produced significantly $(\mathrm{p}<0.05)$ taller plant height and ear height in early season compared with the other genotypes, but statistically similar with 'Ugwuachara' on ear height. However, at late season planting, 'Ugwuachara' genotype gave significantly taller heights than the other genotypes. Stem borer incidence was higher in late planting season when compared to early planting season. The stem borer incidence on the maize genotypes differed significantly in early season, but was nonsignificant at late season. Lower incidence of stem borer with no damage observed on the maize stems at early season planting resulted in the absence of number of exits holes and tunnel damage length on the maize genotypes at earlier season. Yield and yield component traits revealed that the genotypes differed significantly in both seasons with exception of number of ears per plant.

The yield performance of the genotypes at the early season planting was higher than the one obtained at late season planting. The early season had $27.8 \%$ increases in yield over that late season planting. The genotype 'Ugwuachara' gave significantly $(\mathrm{p}<0.05)$ higher grain yield than the other genotypes at late season planting except 'TZE - Y - POP DT STR QPM', while at early season planting 'Ugwuachara' genotype differed significantly $(\mathrm{p}<$ 0.05 ) from the other thirteen genotypes, ranging from 5.40 t/ha to 7.40 t/ha.

\section{Combined analysis of variance for mean square}

The combined statistical analysis for the agronomic traits revealed that season and genotype $\times$ season interaction was significant $(p<0.01)$ for traits like days to tasselling, silking, ear height and plant height for the maize genotypes studied (Table 4). The effect of season on yield and yield components were significantly $(\mathrm{p}<0.01)$ different with ear diameter, ear length, ear weight, hundred grain weight and grain yield. However, season had no significant effect on number of ears per plant and shelling percentage. Season showed highly significant effect $(\mathrm{p}<0.01)$ with stem borer incidence. Furthermore, the effect of genotype $\times$ season interaction was significant $(\mathrm{p}<0.01)$ for agronomic traits, yield and yield component traits with the exception of number of ears per plant.

Correlation coefficient among traits in early and late planting seasons

Days to tasselling and silking produced the highest correlation coefficient at both early and late seasons (Table 5). Even more, in both seasons, ear weight produced the highest correlation coefficient value with grain yield. The early season planting showed that days to tasselling $(\mathrm{r}=$ $0.213)$, silking $(r=0.133)$, plant height $(r=0.132)$, ear height $(r=0.195)$, number of ear per plant $(r=0.19)$, shelling percentage $(r=0.019)$ and hundred grain weight had positive non-significant correlation with grain yield.
Positive significant correlation was observed in ear length $\left(r=0.326^{*}\right)$, ear diameter $\left(r=0.346^{* *}\right)$ and ear weight $\left(\mathrm{r}=0.908^{* *}\right)$. However, stem borer incidence $(\mathrm{r}=$ $0.155)$ had negative non-significant correlation with grain yield.

On the other hand, late season planting showed a negative non-significant correlation with days to tasselling $(\mathrm{r}$ $=-0.167)$, silking $(\mathrm{r}=-0.123)$ and stem borer incidence $(\mathrm{r}=$ $-0.024)$ on grain yield. A positive significant correlation of grain yield was obtained in plant height $\left(r=0.417^{* *}\right)$, ear height $\left(r=0.282^{*}\right)$, ear diameter $\left(r=0.594^{* *}\right)$, ear weight $(r$ $\left.=0.946^{* *}\right)$, hunderd grain weight $\left(r=0.441^{* *}\right)$ and shelling percentage $\left(r=0.440^{* *}\right)$. Ear length and number of ears per plant had a positive non-significant correlation with grain yield.

\section{Path coefficient analysis}

The results of the early season planting showed that days to tasselling, ear height, ear length, number of ear per plant, ear weight and shelling percentage had direct positive effect on grain yield, while direct negative effect were obtained on days to silking, plant height, ear diameter and hundred grain weight (Table 6). Ear weight (0.972) followed by days to tasselling $(0.665)$ exerted the highest direct positive effect on grain yield. Conversely, days to silking $(-0.594)$ and plant height $(-0.316)$ had highest direct negative effect on grain yield. Ear weight that had highest direct positive effect on grain yield exerted positive indirect effects through ear length, ear diameter, hundred grain weights, number of ear per plant, ear height, plant height, and days to tasselling and silking. This trait also showed a negative indirect effect on gain yield through shelling percentage and stem borer incidence. Days to tasselling showed indirect positive contribution of grain yield through days to silking, plant height, ear height, number of ear per plant, ear length, ear diameter and ear weight. The highest positive indirect effect of grain yield were observed on days to tasselling via days to silking $(0.649)$, followed by days to tasselling via ear length (0.426) and ear weight via ear length (0.391).

Under late season planting, the results revealed that days to tasselling, plant height, ear length and number of ears per plant, ear weight, shelling percentage and hundred grain weights had direct positive effects on grain yield. Negative direct effects on grain yield were obtained on days to silking, ear height and ear diameter. Ear weight (0.892) and shelling percentage (0.309) exerted the highest direct positive effect with grain yield. Ear weight showed indirect positive effect on grain yield through plant height, ear height, number of ear per plant, ear length, ear diameter, shelling percentage and hundred grain weights. Ear weight also revealed negative indirect contribution on grain yield through days to tasselling, silking and stem borer incidence. Shelling percentage showed positive indirect effect on grain yield via plant height, ear diameter, ear weight, hundred grains weight and stem borer incidence. Shelling percentage also revealed negative indirect effect on grain yield through days to tasselling, silking, and ear height, number of ear per plant and ear length.

In both seasons, days to tasselling, ear length, ear weight, shelling percentage and stem borer incidence showed direct positive contribution on grain yield. Furthermore, these characters (days to silking, ear diameter and hundred grain weight) showed direct negative effect on grain yield at both seasons. 
Table 3. The agronomic yield and yield components traits of the maize genotypes studied

\begin{tabular}{|c|c|c|c|c|c|c|c|c|c|c|c|c|c|c|}
\hline Genotype & $\begin{array}{l}\text { DST } \\
50 \%\end{array}$ & $\begin{array}{l}\text { DSS } \\
50 \%\end{array}$ & $\begin{array}{l}\text { PHM } \\
(\mathrm{cm})\end{array}$ & $\begin{array}{l}\mathrm{EH} \\
(\mathrm{cm})\end{array}$ & NEPP & $\begin{array}{c}\mathrm{EL} \\
(\mathrm{cm})\end{array}$ & $\begin{array}{l}\text { ED } \\
(\mathrm{cm})\end{array}$ & $\begin{array}{c}\text { EW } \\
(\mathrm{g})\end{array}$ & $\begin{array}{l}\text { SP } \\
(\%)\end{array}$ & $\begin{array}{c}\mathrm{HGW} \\
(\mathrm{g})\end{array}$ & $\begin{array}{l}\text { SBI } \\
(\%)\end{array}$ & EXH & $\begin{array}{l}\text { TDL } \\
(\mathrm{cm})\end{array}$ & $\begin{array}{c}\text { GY } \\
(\mathrm{t} / \mathrm{ha})\end{array}$ \\
\hline \multicolumn{15}{|l|}{ Early season } \\
\hline '99 TZE Y - STR QPM' & 52.00 & 57.10 & 162.90 & 67.90 & 1.00 & 24.90 & 17.47 & 226.80 & 76.67 & 32.50 & 2.5 & Nil & Nil & 6.83 \\
\hline 'AMA TZBR - Y - F2' & 58.67 & 61.20 & 184.00 & 90.80 & 1.07 & 27.90 & 19.73 & 243.20 & 80.67 & 28.90 & 3.27 & Nil & Nil & 7.97 \\
\hline 'BR 9928 DMRSR' & 61.33 & 64.23 & 215.00 & 95.40 & 1.04 & 27.10 & 17.77 & 192.70 & 76.67 & 22.30 & 2.93 & Nil & Nil & 5.4 \\
\hline 'BR 9943 DMRSR' & 56.80 & 59.77 & 207.60 & 104.80 & 1.12 & 26.23 & 18.23 & 255.60 & 79.67 & 31.90 & 1.13 & Nil & Nil & 5.87 \\
\hline 'TZBR COMP - YCIF2' & 57.33 & 59.33 & 210.40 & 114.90 & 1.07 & 27.20 & 18.43 & 250.20 & 68.33 & 32.10 & 1.97 & Nil & $\mathrm{Nil}$ & 6.67 \\
\hline 'TZBR COMP - 2 - YCIF2' & 61.23 & 62.43 & 204.80 & 99.80 & 1.07 & 27.40 & 19.17 & 245.00 & 75.67 & 38.37 & 1.2 & Nil & Nil & 7.4 \\
\hline 'DMR - LSR - Y' & 55.20 & 58.23 & 205.40 & 100.10 & 1.11 & 27.23 & 17.30 & 194.90 & 80.00 & 27.00 & 2.93 & Nil & $\mathrm{Nil}$ & 5.83 \\
\hline 'EV DT - Y - 2000 STR QPM' & 55.33 & 58.33 & 208.60 & 103.00 & 1.00 & 25.47 & 18.33 & 193.60 & 80.00 & 30.00 & 2.07 & Nil & Nil & 5.77 \\
\hline 'TZE - Y - POP DT STR QPM' & 51.67 & 53.57 & 167.00 & 70.60 & 1.01 & 25.07 & 18.40 & 190.30 & 80.00 & 33.50 & 3.7 & Nil & Nil & 5.6 \\
\hline 'TZEE - Y -POR STR QPM CO' & 49.80 & 51.10 & 188.80 & 64.40 & 1.04 & 25.07 & 17.67 & 249.40 & 75.00 & 32.80 & 3.13 & Nil & Nil & 7.47 \\
\hline '2009 TZE OR1 DT STR QPM' & 58.77 & 60.57 & 184.30 & 94.30 & 1.04 & 26.17 & 18.43 & 273.90 & 75.33 & 33.70 & 3.83 & Nil & Nil & 8.53 \\
\hline '2009 TZE OR2 DT STR QPM' & 52.67 & 55.67 & 194.90 & 91.80 & 1.04 & 24.90 & 17.90 & 253.20 & 78.33 & 26.20 & 3.2 & Nil & Nil & 8.07 \\
\hline \multicolumn{15}{|l|}{ 'LANDRACE' } \\
\hline 'EDEM ANI' & 58.43 & 61.43 & 230.50 & 128.30 & 1.06 & 25.87 & 17.73 & 197.80 & 70.33 & 26.00 & 1.93 & Nil & Nil & 4.93 \\
\hline 'ISAKPU' & 59.80 & 62.67 & 191.70 & 108.90 & 1.00 & 26.97 & 19.10 & 225.70 & 65.00 & 26.23 & 2.47 & Nil & Nil & 6.17 \\
\hline 'OBUKPA' & 55.13 & 58.00 & 233.00 & 134.30 & 1.16 & 26.80 & 17.87 & 223.60 & 76.00 & 26.80 & 2.17 & Nil & Nil & 6.53 \\
\hline 'ORBAl' & 65.57 & 67.33 & 257.20 & 143.20 & 1.03 & 29.67 & 18.07 & 233.30 & 76.00 & 32.40 & 2.1 & Nil & Nil & 6.97 \\
\hline 'ORBA2' & 55.87 & 57.90 & 266.90 & 165.50 & 1.14 & 27.50 & 18.47 & 271.80 & 66.67 & 27.60 & 2 & Nil & Nil & 7.63 \\
\hline 'UGWUACHARA' & 67.13 & 70.13 & 245.20 & 147.10 & 1.10 & 31.00 & 19.60 & 316.20 & 75.33 & 31.23 & 0.87 & Nil & Nil & 9.97 \\
\hline 'UMUKASI' & 70.43 & 72.43 & 210.40 & 111.80 & 1.19 & 27.03 & 18.53 & 251.70 & 78.00 & 29.27 & 1.83 & Nil & Nil & 8.33 \\
\hline Mean & 58.06 & 60.60 & 208.90 & 107.20 & 1.07 & 26.81 & 18.43 & 237.50 & 75.46 & 29.94 & 1.71 & Nil & $\mathrm{Nil}$ & 6.94 \\
\hline FLSD 0.05 & 2.07 & 2.47 & 20.41 & 24.02 & NS & 1.65 & 1.07 & 52.15 & 3.75 & 0.80 & 2.38 & Nil & Nil & 2.1 \\
\hline \multicolumn{15}{|l|}{ Late season } \\
\hline '99 TZE Y - STR QPM' & 59.77 & 62.40 & 128.20 & 56.90 & 1.03 & 24.63 & 13.90 & 95.00 & 80.00 & 26.40 & 7.73 & 2.00 & 19.2 & 3.07 \\
\hline 'AMA TZBR - Y - F2' & 71.33 & 73.20 & 148.70 & 82.20 & 1.04 & 22.90 & 14.47 & 102.80 & 78.00 & 23.80 & 5.97 & 5.00 & 22.8 & 3.27 \\
\hline 'BR 9928 DMRSR' & 67.23 & 69.50 & 172.30 & 76.50 & 1.06 & 21.20 & 14.23 & 110.60 & 78.00 & 20.60 & 7.57 & 2.00 & 13.3 & 3.57 \\
\hline 'BR 9943 DMRSR' & 69.10 & 72.40 & 133.80 & 63.70 & 1.21 & 22.27 & 13.30 & 97.80 & 75.00 & 25.80 & 6.97 & 2.33 & 13.9 & 2.9 \\
\hline 'TZBR COMP - YCl' & 63.67 & 66.30 & 162.80 & 75.20 & 1.09 & 26.90 & 14.27 & 113.90 & 70.00 & 26.10 & 7.8 & 4.00 & 23.7 & 3.3 \\
\hline 'TZBR COMP - 2 - YCl' & 67.20 & 69.30 & 221.50 & 123.90 & 1.08 & 26.03 & 15.80 & 126.10 & 77.00 & 31.60 & 6.43 & 4.00 & 39 & 4.17 \\
\hline 'DMR - LSR - Y' & 59.53 & 61.50 & 178.30 & 93.60 & 1.14 & 21.97 & 13.30 & 109.10 & 70.00 & 23.20 & 10.33 & 4.33 & 24.7 & 3.03 \\
\hline 'EV DT - Y - 2000 STR QPM' & 57.23 & 58.83 & 154.30 & 54.70 & 1.06 & 23.13 & 14.67 & 102.10 & 82.00 & 20.17 & 7.7 & 3.67 & 21.9 & 3.47 \\
\hline 'TZE - Y - POP DT STR QPM' & 52.23 & 54.20 & 190.20 & 77.40 & 1.04 & 24.70 & 15.37 & 166.10 & 85.00 & 31.20 & 8.37 & 8.00 & 46 & 6.53 \\
\hline 'TZEE - Y -POR STR QPM CO' & 52.23 & 54.20 & 169.90 & 74.00 & 1.05 & 23.80 & 15.50 & 118.30 & 84.00 & 26.90 & 6.97 & 5.33 & 29.2 & 4.3 \\
\hline '2009 TZE OR1 DT STR QPM' & 60.00 & 64.93 & 170.10 & 79.20 & 1.07 & 26.30 & 15.23 & 151.30 & 73.00 & 28.10 & 5.17 & 3.00 & 30.2 & 4.83 \\
\hline '2009 TZE OR2 DT STR QPM' & 57.53 & 59.50 & 136.20 & 62.00 & 1.07 & 22.40 & 13.33 & 87.80 & 76.00 & 23.10 & 6.83 & 5.67 & 27.4 & 2.57 \\
\hline \multicolumn{15}{|l|}{ 'LANDRACE' } \\
\hline 'EDEM ANI' & 64.43 & 67.50 & 150.10 & 77.80 & 1.07 & 26.27 & 14.80 & 120.60 & 63.00 & 22.00 & 5.4 & 2.33 & 25.2 & 3.4 \\
\hline 'ISAKPU' & 60.67 & 62.30 & 135.80 & 64.20 & 1.03 & 23.07 & 15.30 & 102.00 & 74.00 & 23.20 & 7.2 & 3.00 & 24.4 & 3.04 \\
\hline 'OBUKPA' & 62.43 & 65.00 & 182.20 & 121.60 & 1.23 & 24.37 & 15.30 & 120.00 & 72.00 & 25.60 & 5.73 & 5.67 & 47.3 & 3.6 \\
\hline 'ORBAI' & 64.23 & 67.00 & 188.60 & 91.60 & 1.11 & 24.00 & 15.50 & 151.80 & 82.00 & 24.90 & 6.4 & 3.67 & 28.4 & 5.43 \\
\hline 'ORBA2' & 64.23 & 66.10 & 196.00 & 107.20 & 1.23 & 27.90 & 15.30 & 128.90 & 66.00 & 25.70 & 6.1 & 4.67 & 34.8 & 4.53 \\
\hline 'UGWUACHARA' & 70.30 & 72.30 & 240.90 & 124.30 & 1.10 & 28.67 & 16.00 & 188.90 & 79.33 & 30.40 & 6.17 & 3.67 & 33.9 & 7.23 \\
\hline 'UMUKASI' & 69.23 & 71.20 & 195.40 & 95.20 & 1.27 & 26.07 & 14.10 & 108.30 & 74.00 & 23.80 & 6.37 & 2.00 & 15.9 & 3.27 \\
\hline Mean & 62.74 & 65.14 & 171.30 & 84.30 & 1.12 & 24.45 & 14.77 & 25.45 & 75.86 & 121.10 & 3.91 & 6.91 & 27.4 & 3.92 \\
\hline FLSD 0.05 & 2.13 & 1.92 & 24.09 & 16.14 & NS & 1.88 & 1.44 & 2.43 & 5.39 & 23.79 & NS & NS & NS & 0.84 \\
\hline
\end{tabular}

DST = Days to tasselling, DSS = Days to silking, EH $=$ Ear height, $\mathrm{PHM}=$ Plant height at maturity, ED = Ear Diameter, EL = Ear length, NEPP $=$ Number of ear per plant, $\mathrm{EW}=$ Ear weight, $\mathrm{SP}=$ Shelling percentage, $\mathrm{HGW}=$ Hundred grain weight, $\mathrm{SBI}=$ stem borer incidence, $\mathrm{GY}=$ Grain yield; ${ }^{* *}=$ Highly significant, ${ }^{*}=$ Significant.

Table 4. Combined analysis of variance for the studied maize genotypes

\begin{tabular}{|c|c|c|c|c|c|c|c|c|c|c|c|c|c|}
\hline Factor & $\mathrm{DF}$ & DST & DSS & $\begin{array}{l}\mathrm{EH} \\
(\mathrm{cm})\end{array}$ & $\begin{array}{c}\text { PHM } \\
(\mathrm{cm})\end{array}$ & $\begin{array}{l}\text { ED } \\
(\mathrm{cm})\end{array}$ & $\begin{array}{c}\text { EL } \\
(\mathrm{cm})\end{array}$ & NEPP & $\begin{array}{c}\mathrm{EW} \\
(\mathrm{g})\end{array}$ & $\begin{array}{c}\mathrm{HGW} \\
(\mathrm{g})\end{array}$ & $\begin{array}{l}\text { SP } \\
(\%)\end{array}$ & $\begin{array}{l}\text { SB } \\
(\%)\end{array}$ & $\begin{array}{c}\text { GY } \\
(\mathrm{t} / \mathrm{ha})\end{array}$ \\
\hline Season $(S)$ & 1 & $623.40^{* *}$ & $587.07^{* *}$ & $14967.90^{* *}$ & $40134.4^{* *}$ & $370.80^{* *}$ & $159.15^{* *}$ & $0.04 \mathrm{~ns}$ & $3797237.70^{\circ}$ & $573.53^{* *}$ & NS & $190.46^{* *}$ & $582.95^{* *}$ \\
\hline Genotype (G) & 18 & $159.84^{*}$ & $159.23^{* *}$ & $2845.90^{* *}$ & $3776.10^{* *}$ & $2.61^{* *}$ & $11.54^{* *}$ & $0.19^{*}$ & $3106.50^{* *}$ & $65.58^{* *}$ & $123.92^{* *}$ & $1.25 \mathrm{~ns}$ & $5.65^{* *}$ \\
\hline $\mathrm{G} \times \mathrm{S}$ & 18 & $24.48^{* *}$ & $23.16^{* *}$ & $800.6^{* *}$ & $1321.48^{* *}$ & $1.49^{*}$ & $7.16^{* *}$ & ns & $2305.30^{* *}$ & $8.51^{* *}$ & $37.03^{* *}$ & $0.86 \mathrm{~ns}$ & $4.04^{* *}$ \\
\hline Error & 74 & 1.65 & 2.1 & 150.2 & 177.8 & 0.58 & 1.19 & 0.008 & 670.9 & 1.19 & 7.85 & 3.06 & 1.02 \\
\hline
\end{tabular}

DST = Days to tasselling, DSS $=$ Days to silking, $\mathrm{EH}=$ Ear height, $\mathrm{PHM}=$ Plant height at maturity, ED $=$ Ear Diameter, EL $=$ Ear length, NEPP $=$ Number of ear per plant, $\mathrm{EW}=$ Ear weight, $\mathrm{SP}=$ Shelling percentage, $\mathrm{HGW}=$ Hundred grain weight, $\mathrm{SBI}=$ stem borer incidence, $\mathrm{GY}=$ Grain yield; ${ }^{* *}=$ Highly significant,${ }^{*}=$ Significant. 
572

Table 5. Correlation coefficient analysis on some of the maize traits in early and late planting seasons

\begin{tabular}{|c|c|c|c|c|c|c|c|c|c|c|c|c|}
\hline & $\begin{array}{l}\text { DST } \\
\end{array}$ & DSS & PHM & $\mathrm{EH}$ & NEPP & EL & ED & $\mathrm{EW}$ & SP & HGW & SBI & GY \\
\hline \multicolumn{13}{|l|}{ Early } \\
\hline DST & 1 & & & & & & & & & & & \\
\hline DSS & $0.975^{* *}$ & 1 & & & & & & & & & & \\
\hline PHM & $0.425^{* *}$ & $0.398^{* *}$ & 1 & & & & & & & & & \\
\hline $\mathrm{EH}$ & $0.458^{* *}$ & $0.439^{* *}$ & $0.894^{* *}$ & 1 & & & & & & & & \\
\hline NEPP & 0.179 & 0.149 & 0.253 & $0.291^{*}$ & 1 & & & & & & & \\
\hline EL & $0.640^{* *}$ & $0.629^{* *}$ & $0.538^{* *}$ & $0.570^{* *}$ & 0.173 & 1 & & & & & & \\
\hline ED & $0.309^{*}$ & $0.282^{*}$ & 0.042 & 0.223 & 0.086 & $0.418^{* *}$ & 1 & & & & & \\
\hline EW & 0.182 & 0.117 & 0.234 & $0.286^{*}$ & 0.143 & $0.320^{*}$ & $0.402^{* *}$ & 1 & & & & \\
\hline SP & -0.057 & -0.041 & $-0.292^{*}$ & $-0.38^{* *}$ & 0.034 & -0.122 & -0.065 & -0.175 & 1 & & & \\
\hline HGW & -0.016 & -0.079 & -0.242 & -0.223 & -0.110 & 0.022 & 0.234 & 0.243 & 0.16 & 1 & & \\
\hline SBI & -0.176 & -0.159 & 0.008 & -0.018 & 0.005 & -0.051 & -0.091 & $-0.299^{*}$ & 0.089 & $-0.300^{*}$ & 1 & \\
\hline GY & 0.213 & 0.133 & 0.132 & 0.195 & 0.19 & $0.326^{*}$ & $0.346^{* *}$ & $0.908^{* *}$ & 0.019 & 0.221 & -0.155 & 1 \\
\hline \multicolumn{13}{|l|}{ Late } \\
\hline DST & 1 & & & & & & & & & & & \\
\hline DSS & $0.947^{* *}$ & 1 & & & & & & & & & & \\
\hline PHM & 0.222 & 0.215 & 1 & & & & & & & & & \\
\hline $\mathrm{EH}$ & $0.394^{* *}$ & $0.370^{* *}$ & $0.825^{* *}$ & 1 & & & & & & & & \\
\hline NEPP & $0.310^{*}$ & $0.325^{*}$ & 0.135 & 0.217 & 1 & & & & & & & \\
\hline EL & 0.110 & 0.185 & $0.398^{* *}$ & $0.416^{* *}$ & 0.095 & 1 & & & & & & \\
\hline ED & -0.159 & -0.111 & $0.435^{* *}$ & $0.331^{*}$ & -0.194 & $0.463^{* *}$ & 1 & & & & & \\
\hline EW & -0.065 & -0.022 & $0.498^{* *}$ & $0.375^{* *}$ & 0.082 & $0.321^{*}$ & $0.599^{* *}$ & 1 & & & & \\
\hline SP & $-.0335^{*}$ & $-0.301^{*}$ & 0.007 & -0.225 & $-0.35^{* *}$ & $-0.272^{*}$ & 0.161 & 0.144 & 1 & & & \\
\hline HGW & -0.039 & -0.011 & $0.523^{* *}$ & $0.463^{* *}$ & -0.163 & $0.451^{* *}$ & $0.455^{* *}$ & $0.418^{* *}$ & 0.174 & 1 & & \\
\hline SBI & -0.112 & -0.129 & -0.201 & -0.187 & 0.075 & -0.218 & -0.159 & -0.024 & 0.001 & -0.194 & 1 & \\
\hline GY & -0.167 & -0.123 & $0.471^{* *}$ & $0.282^{*}$ & 0.048 & 0.216 & $0.594^{* *}$ & $0.946^{* *}$ & $0.440^{* *}$ & $0.441^{* *}$ & -0.024 & 1 \\
\hline
\end{tabular}

plant, $\mathrm{EW}=$ Ear weight, $\mathrm{SP}=$ Shelling percentage, $\mathrm{HGW}=$ Hundred grain weight, $\mathrm{SBI}=$ stem borer incidence, $\mathrm{GY}=$ Grain yield, ${ }^{* *}=$ Highly significant, ${ }^{*}=$ Significant.

Table 6. Path coefficient analysis showing the direct and indirect effects on some of the maize traits with yield for early and late planting seasons

\begin{tabular}{|c|c|c|c|c|c|c|c|c|c|c|c|c|}
\hline & $\overline{\text { DST }}$ & DSS & PHM & $\overline{\mathrm{EH}}$ & NEPP & $\mathrm{EL}$ & ED & EW & SP & $\mathrm{HGW}$ & SBI & $\overline{\mathrm{GCY}}$ \\
\hline \multicolumn{13}{|l|}{$\begin{array}{l}\text { Early } \\
\text { season }\end{array}$} \\
\hline DST & 0.665242 & -0.57957 & -0.13448 & 0.076393 & 0.005492 & 0.072025 & -0.03752 & 0.176982 & -0.00997 & 0.000823 & -0.02242152 & 0.213 \\
\hline DSS & 0.648611 & -0.59443 & -0.12593 & 0.073224 & 0.004572 & 0.070787 & -0.03424 & 0.113774 & -0.00717 & 0.004064 & -0.02025581 & 0.133 \\
\hline PHM & 0.282728 & -0.23658 & -0.31642 & 0.149116 & 0.007762 & 0.060546 & -0.0051 & 0.227548 & -0.05107 & 0.012449 & 0.00101916 & 0.132 \\
\hline EH & 0.304681 & -0.26095 & -0.28288 & 0.166797 & 0.008928 & 0.064147 & -0.02708 & 0.278114 & -0.06594 & 0.011472 & -0.00229311 & 0.195 \\
\hline NEPP & 0.119078 & -0.08857 & -0.08005 & 0.048538 & 0.030682 & 0.019469 & -0.01044 & 0.139057 & 0.005946 & 0.005659 & 0.000636975 & 0.19 \\
\hline EL & 0.425755 & -0.37389 & -0.17023 & 0.095074 & 0.005308 & 0.112538 & -0.05076 & 0.311177 & -0.02134 & -0.00113 & -0.00649714 & 0.326 \\
\hline ED & 0.20556 & -0.16763 & -0.01329 & 0.037196 & 0.002639 & 0.047041 & -0.12143 & 0.390916 & -0.01137 & -0.01204 & -0.01159295 & 0.346 \\
\hline EW & 0.121074 & -0.06955 & -0.07404 & 0.047704 & 0.004387 & 0.036012 & -0.04882 & 0.972427 & -0.03061 & -0.0125 & -0.038091112 & 0.908 \\
\hline SP & -0.03792 & 0.024371 & 0.092394 & -0.06288 & 0.001043 & -0.01373 & 0.007893 & -0.17017 & 0.174897 & -0.00823 & 0.01133816 & 0.019 \\
\hline HGW & -0.01064 & 0.04696 & 0.076573 & -0.0372 & -0.00337 & 0.002476 & -0.02842 & 0.2363 & 0.027983 & -0.05144 & -0.03821851 & 0.221 \\
\hline SBI & -0.11708 & 0.094514 & -0.00253 & -0.003 & 0.000153 & -0.00574 & 0.011051 & -0.29076 & 0.015566 & 0.015433 & 0.127395052 & -0.155 \\
\hline Residual & & & & & & & & & & & & 0.090931 \\
\hline \multicolumn{13}{|l|}{$\begin{array}{c}\text { Late } \\
\text { season }\end{array}$} \\
\hline$\overline{\text { DST }}$ & 0.041861 & -0.0517 & 0.007497 & -0.00169 & -0.00471 & 0.001903 & 0.001785 & -0.058 & -0.10382 & 0.000202 & -0.00032014 & -0.167 \\
\hline DSS & 0.039642 & -0.05459 & 0.007261 & -0.00159 & -0.00494 & 0.003201 & 0.001246 & -0.01963 & -0.09329 & 0.000057 & -0.00036874 & -0.123 \\
\hline PHM & 0.009293 & -0.01174 & 0.033772 & -0.00355 & -0.00205 & 0.006887 & -0.00488 & 0.444384 & 0.002169 & -0.00271 & -0.00057455 & 0.471 \\
\hline EH & 0.016493 & -0.0202 & 0.027862 & -0.0043 & -0.0033 & 0.007198 & -0.00372 & 0.334626 & -0.06973 & -0.0024 & -0.00053453 & 0.282 \\
\hline NEPP & 0.012977 & -0.01774 & 0.004559 & -0.00093 & -0.0152 & 0.001644 & 0.002178 & 0.073172 & -0.10971 & 0.000845 & 0.000214385 & -0.048 \\
\hline EL & 0.004605 & -0.0101 & 0.013441 & -0.00179 & -0.00144 & 0.017303 & -0.0052 & 0.28644 & -0.0843 & -0.00234 & -0.00062314 & 0.216 \\
\hline ED & -0.00666 & 0.006059 & 0.014691 & -0.00142 & 0.002949 & 0.008011 & -0.01123 & 0.53451 & 0.049898 & -0.00236 & -0.00045449 & 0.594 \\
\hline EW & -0.00272 & 0.001201 & 0.016818 & -0.00161 & -0.00125 & 0.005554 & -0.00672 & 0.892337 & 0.044629 & -0.00217 & $6.8 \times 10^{-5}$ & 0.946 \\
\hline SP & -0.01402 & 0.016431 & 0.000236 & 0.000968 & 0.005381 & -0.00471 & -0.00181 & 0.128496 & 0.309923 & -0.0009 & $2.8 \times 10^{6}$ & 0.44 \\
\hline HGW & -0.00163 & 0.0006 & 0.017663 & -0.00199 & 0.002478 & 0.007804 & -0.00511 & 0.372997 & 0.053927 & -0.00518 & -0.00055454 & 0.441 \\
\hline SBI & -0.00469 & 0.007042 & -0.00679 & 0.000804 & -0.00114 & -0.00377 & 0.001785 & -0.02142 & 0.00031 & 0.001005 & 0.002858467 & -0.024 \\
\hline Residual & & & & & & & & & & & & 0.009621 \\
\hline
\end{tabular}

DST = Days to tasselling, DSS = Days to silking, EH = Ear height, PHM + Plant height at maturity ED $=$ Ear Diameter, EL = Ear length, NEPP $=$ Number of ear per plant, EW = Ear weight, $\mathrm{SP}=$ Shelling percentage, $\mathrm{HGW}=$ Hundred grain weight, $\mathrm{SBI}=$ Stemborer incidence, GCY = Genotypic correlation coefficient of each traits to yield. 


\section{Discussion}

The weather differences observed during the cropping interval differentiated the two seasons. The significant differences observed among the season in days to tasselling, silking, plant height, ear height, ear diameter, ear length, ear weight, hundred grain weights, stem borer incidence and grain yield indicated that planting season affected these traits. The traits were significant revealed by the differences in genetic makeup of the genotypes which have pre-requisite advantages in breeding programs. Beiragi et al. (2011) reported that planting date had a significant influence on plant height, kernel weight, kernel depth, physiological maturity and total leaf number in maize.

In the present study, plant phenological traits (days to tasselling and silking) showed that they are weather dependent, hence affected the yield of the maize genotypes evaluated at both seasons. In early season planting, a period of short dry spell occurred during the maize genotype establishment at early vegetative growth stage, while this event happened at pre-flowering stage (anthesis/silking) during late season planting. This period of short dry spell that occurred before flowering affected the sink capacity of the plants and its grain filling stage, thus resulted in the lower yield of the maize genotypes at late season planting. The degree of vulnerability of crop to climate variability depends mainly on the stage of development of the crop at the time of weather aberration (Fakorede, 2001). Early flowering observed on the genotypes during early season planting can be attributed to optimum temperature and better environmental condition during the respective period. Bell and Wrights (1998) attributed seasonal effects to difference in soil moisture status, temperature and incident reduction to variable rates of phenological development. Favorable environmental condition at early season helped in possession of higher number of leaves resulting in more photosynthetic activities, which translated to better yield of the maize genotypes at early season, when compared to late season. Increased light interception had been used to improve maize yield (Schoper et al., 1982).

When the period of light interception and photosynthetic activity was prolonged during grain filling, more photosynthates are likely to be translocated to the sink for storage, which will result in higher grain yield. The higher grain filling phase observed in the early season can also account for its higher grain yield over late season planting. Declined soil moisture content and higher evaporative demand during late season planting may have pre disposed the plants to adaptive mechanism where moisture is conserved through stomata closure. Stomata closure often results in low biomass accumulation and subsequently yield. Agele (2006) observed that early season planting performed better in the number of kernels and weights produced per plant than late season planting. Yield decreases in maize had been associated with late planting date (Namaka et al., 2008; Kamal et al., 2009). Alderich et al. (1975) attributed yield reduction in late season planting to insect and disease pressure, heat and moisture stresses especially during the reproductive phase.

Higher incidence of stem borer observed at late season planting could be as a result of environmental conditions which favour growth and development of stem borer. Hordzi and Bochey (2012) reported that the population of stem borer was high during the minor season (late season) than the major season (early season). They noted that increase in the population was as a result of build up from the early season. Furthermore, higher stem borer incidence, number of exits holes and tunnel damage length observed on the genotypes at late season planting contributed to the reduction in grain yield. De Groote (2002) reported that stem borer causes losses ranging between $5-73 \%$ potential yield losses under different agro-ecology conditions.

Ear weight, that showed the greatest direct effect with grain yield at both seasons, implied that well filled grain, heavy or bigger cob contributed more to the yield of maize. The direct effect of traits on grain yield implies its relative importance in increasing grain yield directly and possibility of its exploitation in selection under specific conditions. Several authors have reported contribution of ear weight on the yield of maize (Eleweanya et al., 2005; Henfy, 2011).

The negative direct effect of traits like days to silking, ear diameter and hundred grain weight in both seasons suggested that they counterbalance contributions of other traits to yield. Hence, in late season planting, days to silking took larger period which dragged grain filling stage into moisture stress period and resulted in lower yield. Eleweanya et al. (2005) reported negative direct effect of cob length and ear diameter on maize yield while Majumder et al. (2012) reported negative direct effect of leaf area, flowering shoot percentage and fruit length on mango.

High and positive indirect effects of ear diameter, ear length, ear height and plant height at maturity through ear weight at both seasons are desirable because selection of these traits would lead to increase in ear weight which supports higher grain yield. The negative indirect effect of stem borer incidence through ear weight in both years (0.29076 and -0.02142 in early and late season, respectively) has more magnitude than its direct effect of 0.127395 and 0.002858 in both seasons, respectively. Such higher negative effects counterbalance the positive direct effects as ear weight contributed most to the yield in the maize genotypes, thus reduced yield. The direct positive effect of ear weight, days to tasselling, shelling percentage, and ear length in both seasons indicated that these traits are the major components that affects yield of maize.

Ear weight and days to tasselling, that showed high direct effect on early season planting, contributed to increased grain yield of the maize genotypes, as observed in the early season when compared to the late season. Although at late season days to tasselling and ear weight had positive direct effect, the values were lower when compared to the early season. Favorable weather environment has been noted to influence days to tasselling, thus affects the grain filling stage of the maize genotypes. The higher the grain filling stage is the bigger the ear weight, which translated to higher yield. Henfy (2011) reported high direct positive effect of days to tasselling at early sowing when compared to late sowing.

The low value observed in the residual effects at the two seasons $(11.8 \%$ and $5.0 \%)$ for early and late season, respectively indicated that environmental factors not considered in the study were of less effect to grain yield. 
574

\section{Conclusions}

The study showed that 'Ugwuachara' and 'TZE- Y POP DT STR QPM' genotypes could be selected for late season production because of their significant higher yield and adaptability among the other genotypes studied. Among the traits investigated, environmental conditions exerted less influence on the number of ears per plant and shelling percentage. Early planting season favors growth and yield of maize compared with the late planting season. Ear weight, days to tasselling, shelling percentage and ear length are the major traits that affected grain yield at both seasons. Farmers should adopt planting early for optimum yield of the maize genotypes in derived Savannah agro-ecology.

\section{References}

Agele SO (2006). Weather and seasonal effects on growth, seed yield and solublecarbohydrate concentrations in selected maize cultivars in the humid areas of Nigeria. African Journal of Agricultural Research 1(4):101-110.

Aldrich SR, Scott WO, Leng ER (1975). Modern corn production. $2^{\text {nd }} e d$, A \& L Publications, Champaign III, pp 103-117.

Beiragi MA, Khorasani SK, Shojaei SH, Dadresan M, Mostafavi K, Golbashy M (2011). A study on effects of planting dates on growth and yield of 18 corn hybrids (Zea mays L.). American Journal of Experimental Agriculture 1(3):110-120.

Bell M.J, Wright GC (1998). Groundnut growth and development in contrasting environment. Growth and plant density responses. Experimental Agriculture 34(1):99-112

Bello O B, Ige SA, Azeez MA, Afolabi MS, Abdulmaliq SY, Mahamood J (2012). Heritability and genetic advance for grain yield and its component characters in maize (Zea mays L). International Journal of Plant Research 2(5):138-145.

De Groote H (2002). Maize yield losses from stemborers in Kenya. International Journal of Tropical Insect Science 22(2):89-96.

Dewey DR, Lu KH (1959). A correlation and path coefficient analysis of components of crested wheat grass seed production. Agronomy Journal 51(9):515-518.

Eckert D (1995). Corn production in Ohio. Retrieved 23 Febr 2018 from http: //ohioline. osu. Edu/b472/.Htm

Eleweanya NP, Uguru MI, Eneobong EE, Okocha PI (2005). Correlation and path coefficient analysis of grain yield related characters in maize (Zea mays L.) under umudike conditions of South Eastern Nigeria. Academic Research Journal of Agricultural Science and Research 5(1):19.

Fakorede MAB (2001). Revolutionizing Nigerian agriculture with the golden seed. Inaugural lecture, Obafemi Awolowo University, Ile-Ife.

Henfy M (2011). Genetic parameters and path analysis of yield and its components in corn inbred lines ( Zea mays $\mathrm{L})$ at different sowing dates. Asian Journal ofCropScience 3(3):106-117.
Hordzi WHK, Botchey MA (2012). Some parasitoids of Lepidopterous stem borer pests on maize in Southern Ghana. Bulletin of Environment, Pharmacology and Life Sciences 1(6):77-83.

Ibrahim K, Shamsudin MN, Yacob R, Radan A (2014). Economic impact of climate change on maize production in Northern Nigeria. Trends in Applied Science and Research 9(9):522-533.

Kamara Y, Ekeleme F, Chikoye D, Omioigui LO (2009). Planting date and cultivar effects on grain yield in dry land corn production. Agronomy Journal 101(1):91-98.

Linderholm HW, Walther A, Chen D (2008). Twentieth-century trends in the thermal growing season in the Greater Baltic Area. Climatic Change 87(3-4):405-419.

Majumder DAN, Hassan L, Rahim MA, Kabir MA (2012). Correlation and path coefficient analysis of mango (Mangifera indica $\mathrm{L}$.). Bangladesh Journal of Agricultural Research 37(3): 493-503.

Namaka A, Abubakar IU, Sadik IA, Sharifai AI, Hassas AH (2008). Effect of sowing date and nitrogen level on yield and yield components of two extra early maize varieties (Zea mays L.) in Sudan savanna of Nigeria. Journal of Agricultural and Biological Science 3(2):1-5.

Nielson RL, Thomison PR, Brown GA, Halter AL, Wells J, Wuethricc KL (2002). Delayed planting date effects on flowering and grain maturation of corn. Agronomy Journal 94(3):549-558.

Norwood CA (2001). Dryland corn production in Western Kansas: effect of hybrid maturity, planting date and plant population. Agronomy Journal 93(3):540-547.

Obi IU (1981). Identified major disease hazards of maize production in Southern Nigeria. Der Tropenlandwirt - Journal of Agriculture in the Tropics and Subtropics 82(2):137-144.

Olakojo SA, Akinlosotu TA (2004). Comparative study of storage methods of maize grains in South Western Nigeria. African Journal of Biotechnology3(7):362-365.

Olawuyi OJ, Odebode AC, Alfar A, Olakojo SA, Adesoye AI (2010). Performance of maize genotypes and arbuscular mycorrhizal fungi in Samara district of South West region of Doha Qatar. Nigerian Journal of Mycology 3(1):86-100.

Rani GU, Rao VS, Ahmad ML, Rao KLN (2017). Character association and path coefficient analysis of grain yield and yield components in maize (ZeamaysL.). International Journal of Current Microbiology and Applied Sciences 6(12):4044-4050.

Tyagi SD, Khan MH (2010). Studies on genetic variability and inter relationship among different traits in Microsperma lentil (Lens culinaris Medik). Journal of Agriculture Biotechnology and Sustainable Development 2(1):15-20.

Uguru MI, Baiyeri KP, Abba SC (2011). Indicators of climate change in derived Savannah niche on Nsukka, South Eastern Nigeria. AgroScience 10(1):17-26.

Wright S (1934). The method of path coefficients. The Annals of Mathematical Statistics 5(3):161-215. 